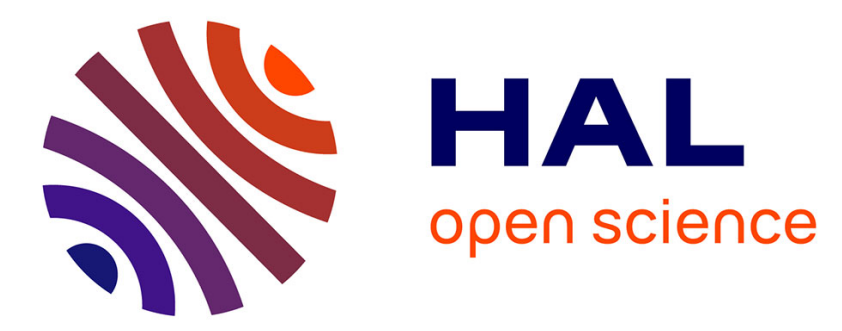

\title{
$35 \mathrm{Cl} \mathrm{NQR}$ and calorimetric reinvestigation of the incommensurate phase of bis(4-chlorophenyl) sulfone: evidence for no lock-in transition
}

J. Etrillard, B. Toudic, M. Bertault, J. Even, M. Gourdji, A. Péneau, L. Guibé

\section{- To cite this version:}

J. Etrillard, B. Toudic, M. Bertault, J. Even, M. Gourdji, et al.. 35Cl NQR and calorimetric reinvestigation of the incommensurate phase of bis(4-chlorophenyl) sulfone: evidence for no lock-in transition. Journal de Physique I, 1993, 3 (12), pp.2437-2449. 10.1051/jp1:1993255 jpa-00246878

\section{HAL Id: jpa-00246878 https://hal.science/jpa-00246878}

Submitted on 1 Jan 1993

HAL is a multi-disciplinary open access archive for the deposit and dissemination of scientific research documents, whether they are published or not. The documents may come from teaching and research institutions in France or abroad, or from public or private research centers.
L'archive ouverte pluridisciplinaire HAL, est destinée au dépôt et à la diffusion de documents scientifiques de niveau recherche, publiés ou non, émanant des établissements d'enseignement et de recherche français ou étrangers, des laboratoires publics ou privés. 


\title{
${ }^{35} \mathrm{Cl}$ NQR and calorimetric reinvestigation of the incommensurate phase of bis(4-chlorophenyl) sulfone : evidence for no lock-in transition
}

\author{
J. Etrillard ( $\left.{ }^{1}\right)$, B. Toudic $\left({ }^{1}\right)$, M. Bertault $\left({ }^{1}\right)$, J. Even $\left({ }^{1}\right)$, \\ and \\ M. Gourdji $\left({ }^{2}\right)$, A. Péneau $\left({ }^{2}\right)$, L. Guibé $\left({ }^{2}\right)$ \\ (1) Groupe Matière Condensée et Matériaux, U.R.A. au C.N.R.S. 040804, Université de \\ Rennes I, Bâtiment B11, Campus de Beaulieu, 35042 Rennes Cedex, France \\ (2) Institut d'Electronique Fondamentale, U.R.A. au C.N.R.S. 0022, Université Paris XI, \\ Bâtiment 220, 91405 Orsay Cedex, France
}

(Received 8 March 1993, accepted in final form 3 August 1993)

\begin{abstract}
Cl} \mathrm{NQR}$ and calorimetric measurements in BCPS have been reinvestigated between $77 \mathrm{~K}$ and room temperature $(\mathrm{NQR})$ and between $100 \mathrm{~K}$ and $180 \mathrm{~K}\left(C_{\mathrm{p}}\right)$. It is shown that, in contradiction with conclusions found in literature, there is no lock-in transition at $115 \mathrm{~K}$. These new results are in agreement with those obtained with other experimental techniques: Raman scattering, X-ray diffraction, neutron diffraction and proton NMR; the latter indicate that the incommensurate phase, appearing on cooling at $150 \mathrm{~K}$, persists down to $4.2 \mathrm{~K}$, at least. The shape of the ${ }^{35} \mathrm{Cl} \mathrm{NQR}$ spectrum in the BCPS incommensurate phase shows that the frequency of a chlorine nucleus is a function of the order parameter, through linear and quadratic terms.
\end{abstract}

\section{Introduction.}

For about fifteen years, a great amount of experimental and theoretical studies concerning the structure and dynamics of incommensurate systems have been reported [1-4]. The collective aspect of the dynamics has been studied by coherent neutron scattering and light scattering and is now rather well understood, even in quite complex systems [1]. Resonance methods, such as NMR, NQR or EPR, have also been very fruitful for the analysis of the different aspects involved in these structures $[1,5,6]$. The resonance lineshape gives specific informations on the local nature of the modulation wave whereas spin-lattice relaxation measurements yield the spectral density of the corresponding excitation modes. Some fundamental questions still remain open concerning especially the influence of the characteristic excitations on local properties : modification of the state density, Debye-Waller factor and heat capacity.. Another point is the analysis of the evolution of the lattice dynamics in the incommensurate phase when going from a sinusoidal regime to a soliton-like one. The study of these questions requires a compound where the incommensurate phase exists over a large temperature region and, even 
better, persists down to lowest temperatures. There are few examples of structurally incommensurate crystals where this is indeed the case and they always concern purely displacive phase transitions $\left(\mathrm{ThBr}_{4}, \mathrm{ThCl}_{4}\right.$, biphenyl, ..). In biphenyl and $\mathrm{ThBr}_{4}$, this property allows the direct observation of the phason branch by coherent neutron scattering because of the low damping of these excitations at low temperature [7, 8]. In biphenyl, the appearance of satellites up to the third order in phase III below $20 \mathrm{~K}$ was explained by higherorder components in the modulation wave. Compounds from the $\mathrm{A}_{2} \mathrm{BX}_{4}$ family, on the opposite, do not remain incommensurate down to the lowest temperatures but several results, often obtained by resonance techniques $[1,4]$, clearly indicate an evolution to non-sinusoidal regimes with soliton-like character in these compounds : unfortunately the large damping of the excitations does not permit direct analysis in these cases.

In this paper, we study a molecular compound in which an incommensurate phase has been found rather recently upon cooling below $150 \mathrm{~K}$ : bis(4-chlorophenyl) sulfone or BCPS ; we were led to this study because contradictory results are found in literature concerning the incommensurate phase in this compound. Let us first consider the major features of BCPS and then the contradictory results.

At room temperature, BCPS crystallizes in a monoclinic structure of space group $\mathrm{C} 2 / \mathrm{c}$ with two molecules per unit cell. The molecule, $\left(\mathrm{ClC}_{6} \mathrm{H}_{4}\right)_{2} \mathrm{SO}_{2}$, is made of two chlorophenyl groups connected to a sulfur atom located on the twofold symmetry axis of the molecule (Fig. 1); the dihedral angle between each benzene ring and the $\mathrm{Cl}-\mathrm{S}-\mathrm{Cl}$ plane is about $84^{\circ}$. Following the intensities of the first-order satellites reflections obtained by $X$-ray [9] and neutron [10] diffraction, it seems that the incommensurate phase exists from $150 \mathrm{~K}$ down to the lowest studied temperature $(13 \mathrm{~K})$. Direct observation of rather strong higher-order satellites by neutron diffraction below about $110 \mathrm{~K}$ [10] is explained by a departure from a sinusoidal regime in the incommensurate phase. Raman scattering clearly shows a soft mode that the authors assumed to be related to a twisting of the phenyl groups [11] and has an exceptionally low damping [12]. Some analogies with biphenyl were then considered as biphenyl is also a molecular compound which presents a second order displacive incommensurate phase with an instability associated to a soft internal degree of freedom. A crystallographic study of the BCPS incommensurate phase is now being made [13] and will inform us on the nature of the

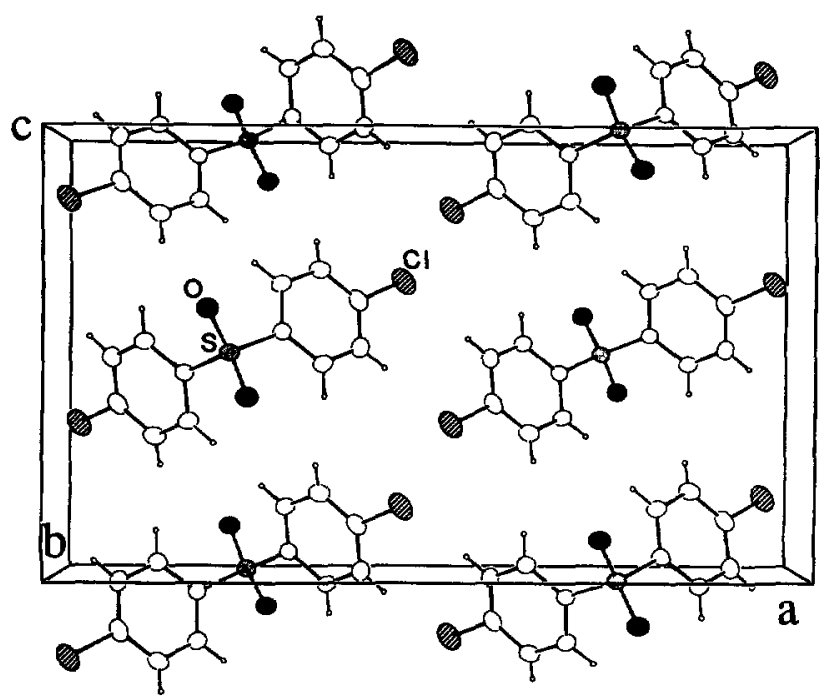

Fig. 1. - Crystalline structure of bis(4-chlorophenyl) sulfone. 
involved motion. As in biphenyl [14], proton NMR measurements also led to the existence of a gapless phason in the incommensurate phase of BCPS [15].

Concerning the extension of the incommensurate phase, results from ${ }^{35} \mathrm{Cl} \mathrm{NQR}$ spectroscopy and reported in two independently published papers $[16,17]$ would indicate a lock-in transition at $115 \mathrm{~K}$. Such a transition was also suggested by AC-calorimetry measurements showing a $C_{\mathrm{p}}$ anomaly at this temperature [18]. In order to clarify the contradiction between the mentioned lock-in transition at $115 \mathrm{~K}$ and the persistence of the incommensurate phase down to the lowest studied temperature, we have reinvestigated the thermodynamic behaviour of this transition. The second part of this paper deals with an experimental reanalysis of the ${ }^{35} \mathrm{Cl} \mathrm{NQR}$ results in this compound.

\section{Differential scanning calorimetry study.}

2.1 EXPERIMENTAL. - Bis(4-chlorophenyl) sulfone was purchased from Aldrich-Chemicals and next purified by recrystallization from benzene solution and zone refining ; single crystals were grown using two techniques, the Bridgman method and crystallization from a benzene solution. The calorimetric measurements were performed in the temperature range extending from $100 \mathrm{~K}$ to $180 \mathrm{~K}$ with a Perkin-Elmer DSC 7 differential scanning calorimeter controlled by a Digital DEC Station 425C micro-computer for data acquisition and processing. The measurement head was immersed in liquid nitrogen and flushed with dry helium gas. The temperature calibration of the instrument was made against the transition temperature $(186.1 \mathrm{~K})$ and melting point $(279.9 \mathrm{~K})$ of cyclohexane [19]. Energy was calibrated from the enthalpy of the phase transition of cyclohexane $\left(79.52 \mathrm{~J} . \mathrm{g}^{-1}\right)$. The calibration exactness was then checked against a sapphire single crystal plate of about $130 \mathrm{mg}$ using the aluminium oxide heat capacity standards at 1 atmosphere [20]. Single crystal samples of BCPS cut from a large crystal and weighting from $60 \mathrm{mg}$ to $80 \mathrm{mg}$, were carefully encapsuled in $50 \mu \mathrm{l}$ aluminium pans in order to ensure good heat transfer. The specific heat measurements were carried out at $5 \mathrm{~K} \cdot \mathrm{min}^{-1}$ and $10 \mathrm{~K} \cdot \mathrm{min}^{-1}$ rates on crystals grown from the melt and benzene solution. Only results obtained with the $10 \mathrm{~K} \cdot \mathrm{min}^{-1}$ scan rate are shown in figures 2 and 3, because with $5 \mathrm{~K} \cdot \mathrm{min}^{-1}$ results are quite similar though a little more noisy.

2.2 RESULTS AND DISCUSSION. - The specific heat curves recorded at increasing temperature in the temperature range $100 \mathrm{~K}-180 \mathrm{~K}$ for the Bridgman and the benzene solution grown crystals are shown in figure 2. Apart a small difference discussed below, the two samples present qualitatively similar behaviours: considering decreasing temperature, a single small endothermic anomaly with a rapid increase around $150 \mathrm{~K}$ and a smooth decay spreading over about $40 \mathrm{~K}$. The fundamental difference between this result that appears in literature [18] is the absence, in the present study, of any anomaly around $115 \mathrm{~K}$. As further discussed below, this is a strong argument for the nonexistence of a lock-in transition in BCPS at this temperature. The $C_{\mathrm{p}}$ anomaly beginning at $150 \mathrm{~K}$ clearly results from the second order incommensurate phase transition. It is worth mentioning that the shape of the anomaly depends on the method of preparation of the sample. Whereas there is a nice typical signature for the Bridgman grown single crystal giving $T_{1}=150 \mathrm{~K}$, the signature is less sharp for the solution grown crystal ; the transition temperature is then more difficult to define and is around $147 \mathrm{~K}$. This actually shows the important role which impurities or crystalline defects may have on the incommensurate phase transition and perhaps also explains the difference with the previous thermodynamic study. The excess of $C_{\mathrm{p}}$ is obtained from substraction of the normal non transitional heat capacity which is described by an extrapolated seventh order polynomial curve following usual procedure. The result is shown in figure 3, for the Bridgman grown single crystal. The values of the enthalpy and the entropy of the transition are respectively $52 \mathrm{~J} . \mathrm{mole}^{-1}$ and 


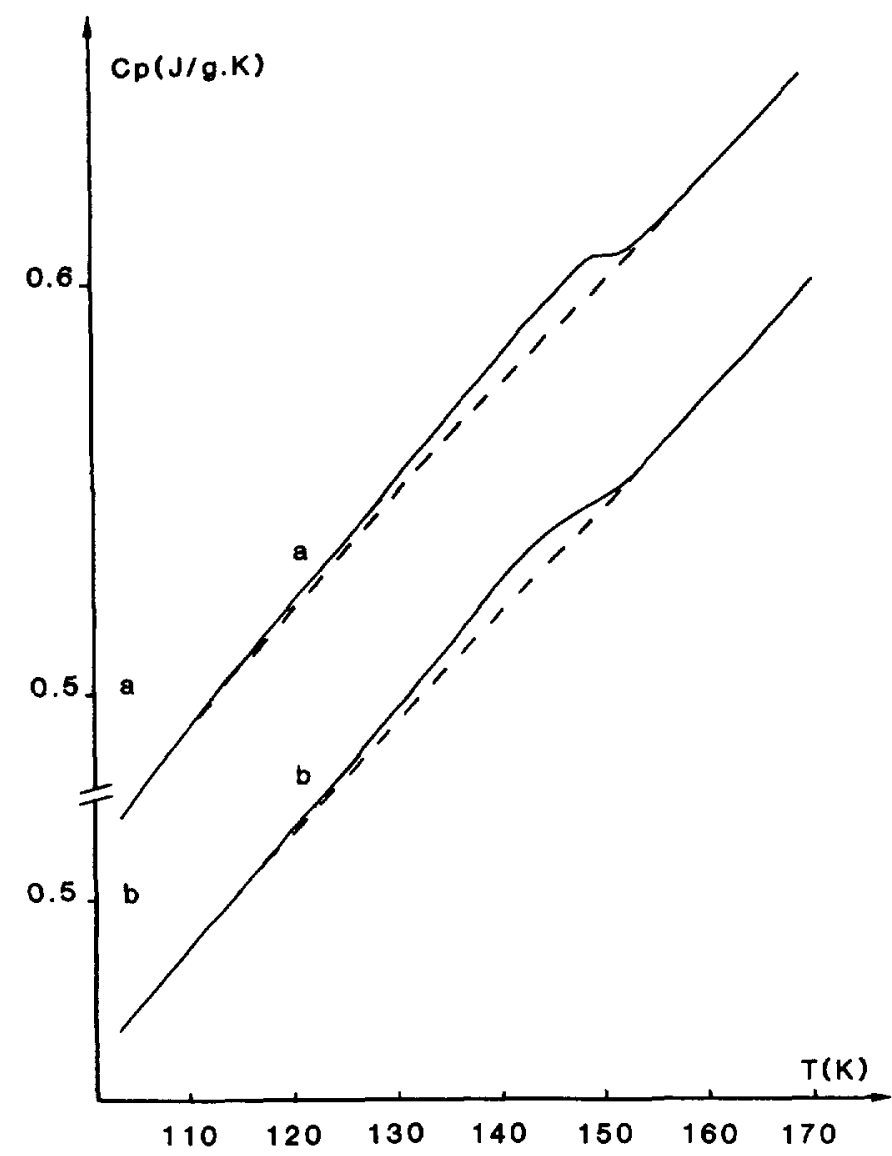

Fig. 2. - Specific heats measured on BCPS single crystals grown a) by the Bridgman method and b) from benzene solution.

$0.38 \mathrm{~J} . \mathrm{mole}^{-1} \cdot \mathrm{K}^{-1}$; they are both a little larger $(20 \%)$ than previously reported [18]. The entropy of the transition remains much smaller than $R . \ln (2)$ as this is the case for all displacive structural phase transitions.

\section{3. ${ }^{35} \mathrm{Cl}$-nuclear quadrupole resonance study.}

3.1 EXPERIMENTAL. - Investigations were performed at the I.E.F. on a laboratory made pulsed NQR spectrometer [21]. The power stage of this spectrometer is now a commercial $1 \mathrm{~kW}$ peak wideband rf power amplifier (Kalmus 166LP), and an usual value for a $90^{\circ}$ pulse width is 16 microsecondes with a $14.5 \mathrm{~mm}$ inner diameter, 5 turn coil. As the free induction decay signal is mixed with the receiver recovery signal, the echo following a standard $90^{\circ}-\tau$ $180^{\circ}$ pulse sequence was recorded. When the spectrum is broad, its shape is obtained from the plot, on the same diagram, of the echo signals recorded at different frequencies [22] : the horizontal position of each echo signal on the diagram is proportional to the spectrometer frequency and the line joining the points of maximum amplitude of each echo is an 


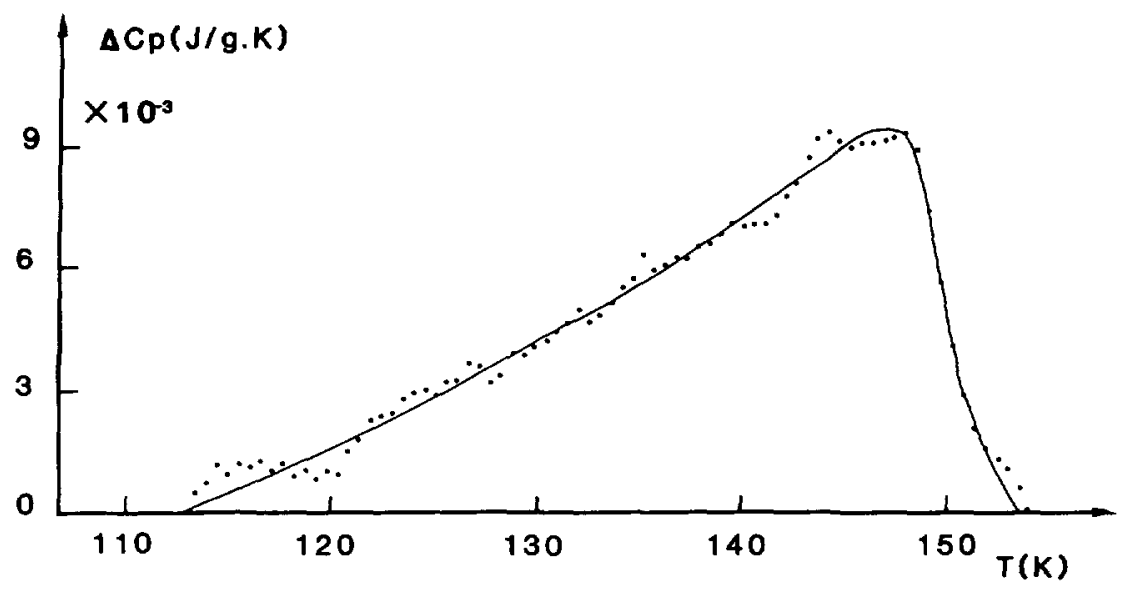

Fig. 3. - Specific heat excess observed in the Bridgman grown BCPS single crystal.

approximation of the shape of the spectrum, or spectral function $f(v)$. Most of the NQR work was done on a large polycrystalline sample of BCPS (Aldrich), recrystallized from the melt, about $14 \mathrm{~mm}$ in diameter and $3 \mathrm{~g}$ in weight. Some spectra were also recorded from the large and high-quality single crystal (diameter: $13.5 \mathrm{~mm}$, weight: $2.6 \mathrm{~g}$ ) which gave the $\Delta C_{\mathrm{p}}$ shown in figure 3.

3.2 NQR SPECTRUM. - Above $150 \mathrm{~K}$, in the high temperature phase of BCPS, there is only one crystallographical site for the chlorine atoms resulting from the molecule and crystal symmetries; thus one half of the molecule suffices to generate the four molecules of the unit cell by symmetry operations. Accordingly, a single NQR resonance line is observed.

Below $150 \mathrm{~K}$, the spectrum is broad and recorded as described in 3.1. Spectra recorded at different temperatures below $150 \mathrm{~K}$ are shown in figure 4; upon cooling, the spectrum broadens steadily and its shape changes from that of a bell to a more complicated one with three resolved maxima separated by two valleys. This structure persists down to liquid nitrogen temperature. The spectra recorded with the polycrystalline and single crystal samples at $77 \mathrm{~K}$ are presented in figure 5: they are quite similar which shows that the quality of the polycrystalline sample used for most of this work is quite satisfactory. At this temperature, the width of the spectrum is about $300 \mathrm{kHz}$. Such a spectral distribution is typical of incommensurate phases where no atom occupies the same position as any homologous atom in another crystal cell of the crystal. The spectra presented in figure 4 show a continuous evolution from $150 \mathrm{~K}$ to $90 \mathrm{~K}$ and no clear difference appears between the spectra at $110 \mathrm{~K}$ and $120 \mathrm{~K}$ which could suggest a transition occurring between these two temperatures, in particular at $115 \mathrm{~K}$; moreover, no narrowing of the resonance peaks leading to a discrete spectrum, as expected for a commensurate phase, is observed.

In order to understand the discrepancy between the present results and those already published [16, 17], we have plotted in figure 6 the experimental maxima recorded by us (crosses) and Nakayama et al. [17] (closed circles). It seems that for temperatures above $115 \mathrm{~K}$, these authors recorded the low frequency and central peaks of the spectrum, whereas for temperatures below $115 \mathrm{~K}$ they have recorded the low and high frequency peaks; the change in the peaks actually recorded, above and below $115 \mathrm{~K}$, leads to the jump in the $v(T)$ curve they report. This may result from a low signal-to-noise ratio or from some 

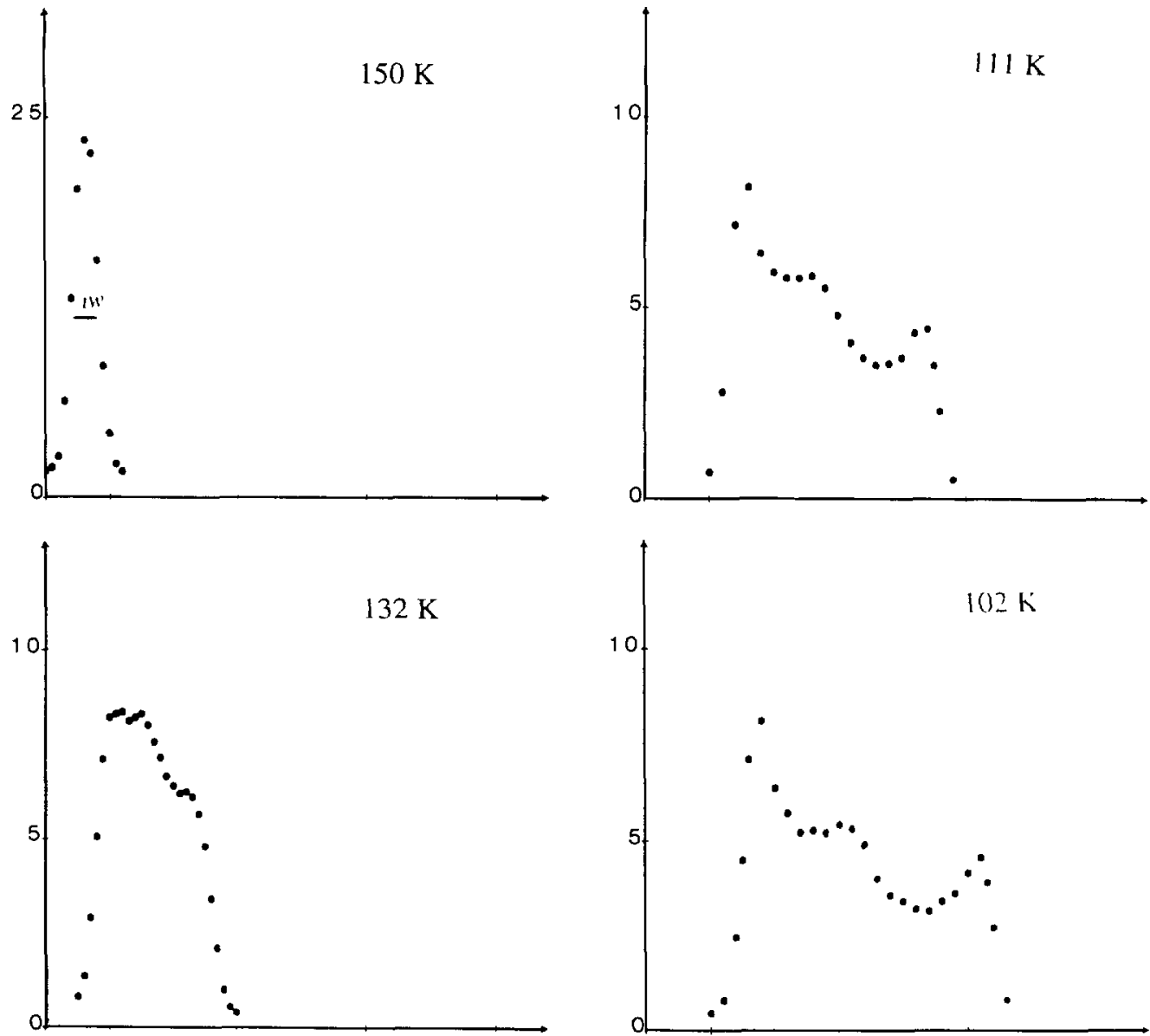

$f(v)$

(a. u.)

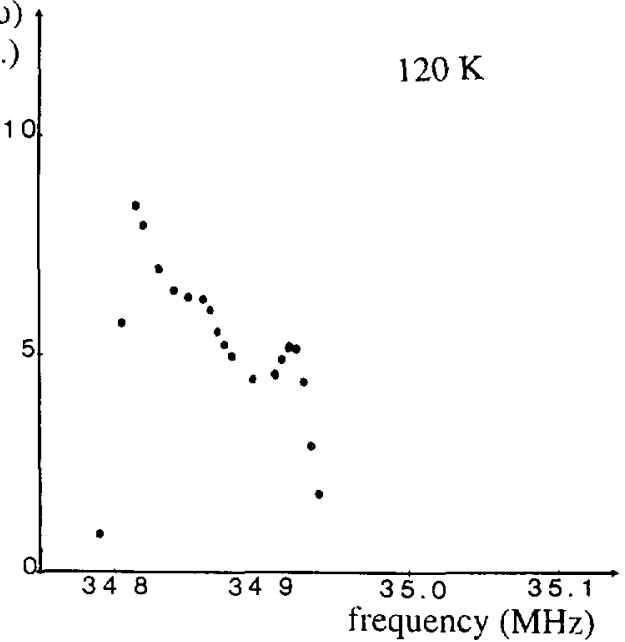

$\mathrm{f}(\mathrm{v})$

(a. u.)

$91 \mathrm{k}$

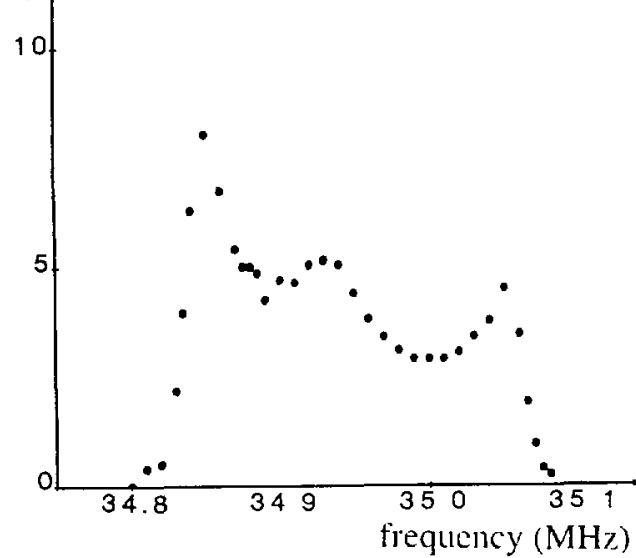

Fig. 4. - Temperature evolution of the ${ }^{35} \mathrm{Cl} \mathrm{NQR}$ recorded spectrum in the BCPS incommensurate phase. The horizontal bar $i w$ in the $150 \mathrm{~K}$ diagram shows the $18 \mathrm{kHz}$ instrumental width of the spectrometer response. It is the same for all the spectra. 


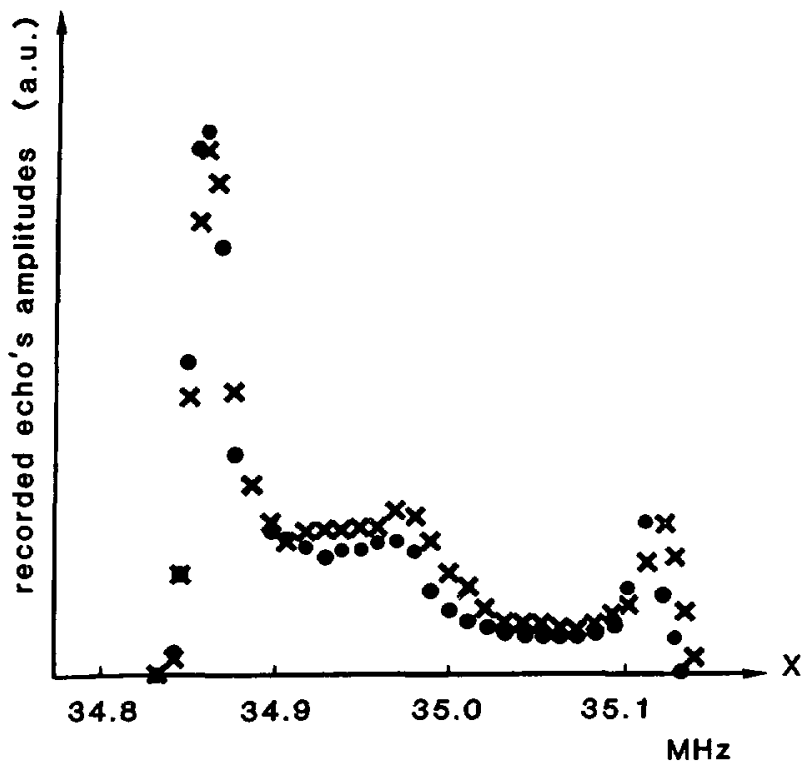

Fig. 5. - Comparison of the ${ }^{35} \mathrm{Cl} \mathrm{NQR}$ spectra in polycrystalline Bridgman grown $(\times)$ single crystal $(\bullet)$ samples of BCPS at $77 \mathrm{~K}$. For technical reasons, phase detection was not used here and the receiver response is nonlinear. Though the single crystal sample gives a stronger signal, the two spectra are drawn with similar amplitude for the sake of convenient comparison.

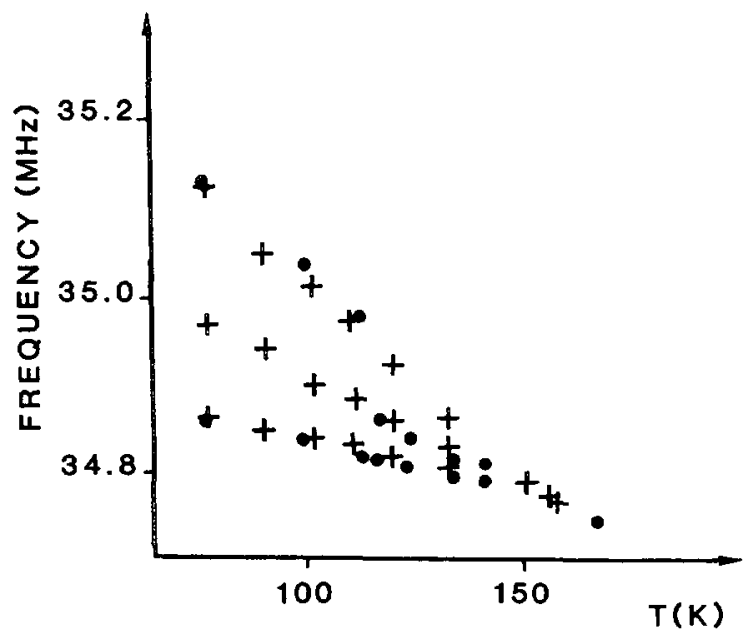

Fig. 6. - Temperature evolution of the different maxima in the BCPS spectra recorded in the present work $(+)$ compared to the results in reference $[16](\bullet)$.

difference in the spectrum shape related to the sample preparation. Indeed, in the temperature range where the BCPS incommensurate phase is studied here, the frequencies of the maxima in the resonance curve vary smoothly with temperature and Nakayama's data can be reinterpreted consistently with the present investigation. The present reinterpretation of the data thus excludes any lock-in transition in this compound down to $77 \mathrm{~K}$. In this study, the shape of the 
recorded spectra confirms the incommensurate character of the BCPS phase appearing below $150 \mathrm{~K}$. It is possible to discuss the results obtained further and propose a tentative model of the temperature dependence of the NQR spectrum. Such an analysis would be useful because the electric field gradient, and also the NQR frequencies, are related to the crystalline structure and constitute parameters to monitor the incommensurate distortion in the crystal.

In the case of a sinusoidal one-dimensional static modulation, the special shape of the resonance line with three maxima, indicates that the NQR frequency of a chlorine nucleus is function of its displacement $u$ with linear and quadratic terms [23] :

$$
v=v_{0}+a_{1} u+a_{2} u^{2}
$$

where $v_{0}$ is the resonance frequency in the high temperature phase. The line shape can be derived from this equation by introducing the modulation :

$$
u=A \cos [\Phi(x)]
$$

where $\Phi(x)=\Phi_{0}+q_{\mathrm{s}}, x$ and $q_{\mathrm{s}}$ is the modulation wave vector. Therefore, the resonance frequency becomes :

$$
v=v_{0}+v_{1} \cos [\Phi(x)]+v_{2} \cos ^{2}[\Phi(x)],
$$

with $v_{1}=a_{1} A$ and $v_{2}=a_{2} A^{2} ; A$ is expected to vary as $\left(T_{\mathrm{I}}-T\right)^{\beta}$, where $\beta$ is the order parameter exponent. The spectral function, which is defined as $f(v)=$ constant $/(|\mathrm{d} v / \mathrm{d} x|)$ has two or three singularities according to the respective values of $v_{1}$ and $v_{2}$, functions of the temperature [23]:

When $\left|v_{2}\right| \leqslant\left|v_{1} / 2\right|$, there are two singularities :

$$
v_{\mathrm{a}}=v_{0}+v_{1}+v_{2} \text { and } v_{\mathrm{b}}=v_{0}-v_{1}+v_{2} .
$$

When $\left|v_{2}\right|>\left|v_{1} / 2\right|$, a third singularity appears at :

$$
v_{c}=v_{0}-v_{1}^{2} /\left(4 v_{2}\right) \text {. }
$$

These singularities correspond to the extrema of the function (3). When three singularities are present, one gets used to decompose the calculation of the spectral function between each extremum and called respectively $g\left(\varphi_{+}\right)$and $g\left(\varphi_{-}\right)[23]$ :

$$
f(v)=\left\{\begin{array}{lll}
g\left(\varphi_{+}\right) & \text {for } & v_{\mathrm{b}}<v \leqslant v_{\mathrm{a}} \\
g\left(\varphi_{-}\right)+g\left(\varphi_{+}\right) & \text {for } & v_{\mathrm{c}} \leqslant v \leqslant v_{\mathrm{b}}
\end{array}\right.
$$

where

$$
g(\varphi)=\frac{\text { constant }}{\left|\sin (\varphi) \cdot\left[v_{1}+2 v_{2} \cdot \cos (\varphi)\right]\right|},
$$

with

$$
\cos \left(\varphi_{ \pm}\right)=\frac{-v_{1} \pm \sqrt{v_{1}^{2}+4\left(v-v_{0}\right) v_{2}}}{2 v_{2}}
$$

Parameters $v_{0}, v_{1}, v_{2}, T_{1}$ and $\beta$ are determined by fitting the frequencies of the peaks in the experimental spectra. However, the response of the spectrometer, acting as a convolution of the physical spectrum by the spectrometer response function, brings some perturbations to the recorded spectrum : i) the top of asymmetrical peaks is slightly shifted towards the smallest slanted side of the peak; ii) the intensity of the peaks with respect to the valleys is reduced; iii) the noise is reduced. To obtain more accurate values of the frequencies of the peaks, a 
deconvolution [24] of the spectra was performed before determining these frequencies. The Bayes algorithm was used taking an $18 \mathrm{kHz}$ half-height wide Gaussian for the deconvolution function. This instrumental function was determined by taking the Fourier transform of the echo given by a sample which has a flat spectrum over a frequency range much greater than the instrumental function, as it is the case in BCPS between the spectrum maxima at the lowest temperature studied $(77 \mathrm{~K})$; its width was then confirmed from the plot of a sharp NQR line, the one of BCPS at room temperature, recorded as done for broad spectra. A least square fit using the frequencies of the maxima in the deconvoluted spectra leads to the following parameters values, the transition temperature being $T_{\mathrm{I}}=150 \mathrm{~K}$ :

$$
\begin{aligned}
& \beta=0.5 \\
& v_{1}=7.25 \cdot\left(T_{I}-T\right)^{\beta} \mathrm{kHz} \\
& v_{2}=2.14 \cdot\left(T_{\mathrm{I}}-T\right)^{2 \beta} \mathrm{kHz} .
\end{aligned}
$$

For the temperature dependence of $v_{0}$ in (3), resulting from the averaging effect of the molecular angular oscillations and described by the Bayer's theory, we used the approximation [25]:

$$
v_{0}=a T+b
$$

with $a=-1.0 \mathrm{kHz} \cdot \mathrm{K}^{-1}$ and $b=34.946 \mathrm{MHz}$.

This set of values is different from that found above $T_{\mathrm{I}}$, where the temperature dependence of the frequency is quite linear :

$$
a=-2.92 \mathrm{kHz} \cdot \mathrm{K}^{-1} \text { and } b=35.22 \mathrm{MHz} \text {. }
$$

The difference between the $a$ values, above and below the transition, is an indication of a corresponding difference in the crystal dynamics. This is consistent with the change observed in the intensity of the Bragg $(0,2,0)$ reflection and the slopes of the temperature dependence of the crystal parameter $a$ as shown in figures 1 and 2 of reference [10].

Concerning the determination of $\beta$, the results of the fitting procedure appear to be very sensitive to the extension of the temperature range considered below $T_{1}$. Changes of more than 0.15 were found on the values of $\beta$ according to the temperature range considered in this fitting procedure. A value of $\beta$ close to 0.5 was found, using the whole temperature range, and is used henceforth. This value, which corresponds to the mean field approximation is consistent with the results of others methods of investigation : X-ray diffraction [9], neutron diffraction [10] and Raman scattering $[11,12]$.

With the determined parameters and the $18 \mathrm{kHz}$ wide Gaussian instrumental response as convoluting function, spectra at $132 \mathrm{~K}, 120 \mathrm{~K}, 111 \mathrm{~K}, 102 \mathrm{~K}$ and $91 \mathrm{~K}$ have been calculated (Fig. 7). They are relatively good agreement with experimental recorded spectra shown in figure 4 .

A possible difference between theoretical and experimental curves may be the non validity of the sinusoidal model for the static modulation when going further below $T_{\mathrm{I}}$, especially below $100 \mathrm{~K}$. The evolution of the modulation towards a nonsinusoidal regime is suggested by the analysis of high order satellites in neutron diffraction experiments [10], and the discussion of X-ray diffraction measurements taking these satellites into account [13]. In this last case, better crystallographic description is obtained by introducing second harmonic in the modulation. Calculation of the resulting NQR spectra would now be quite complex because much more parameters have to be introduced (weight and phase of the second harmonic relative to the first one). The effect of this so-called soliton regime on NQR spectra has been qualitatively discussed [23]. The modulation is described as commensurate quasimacroscopic 


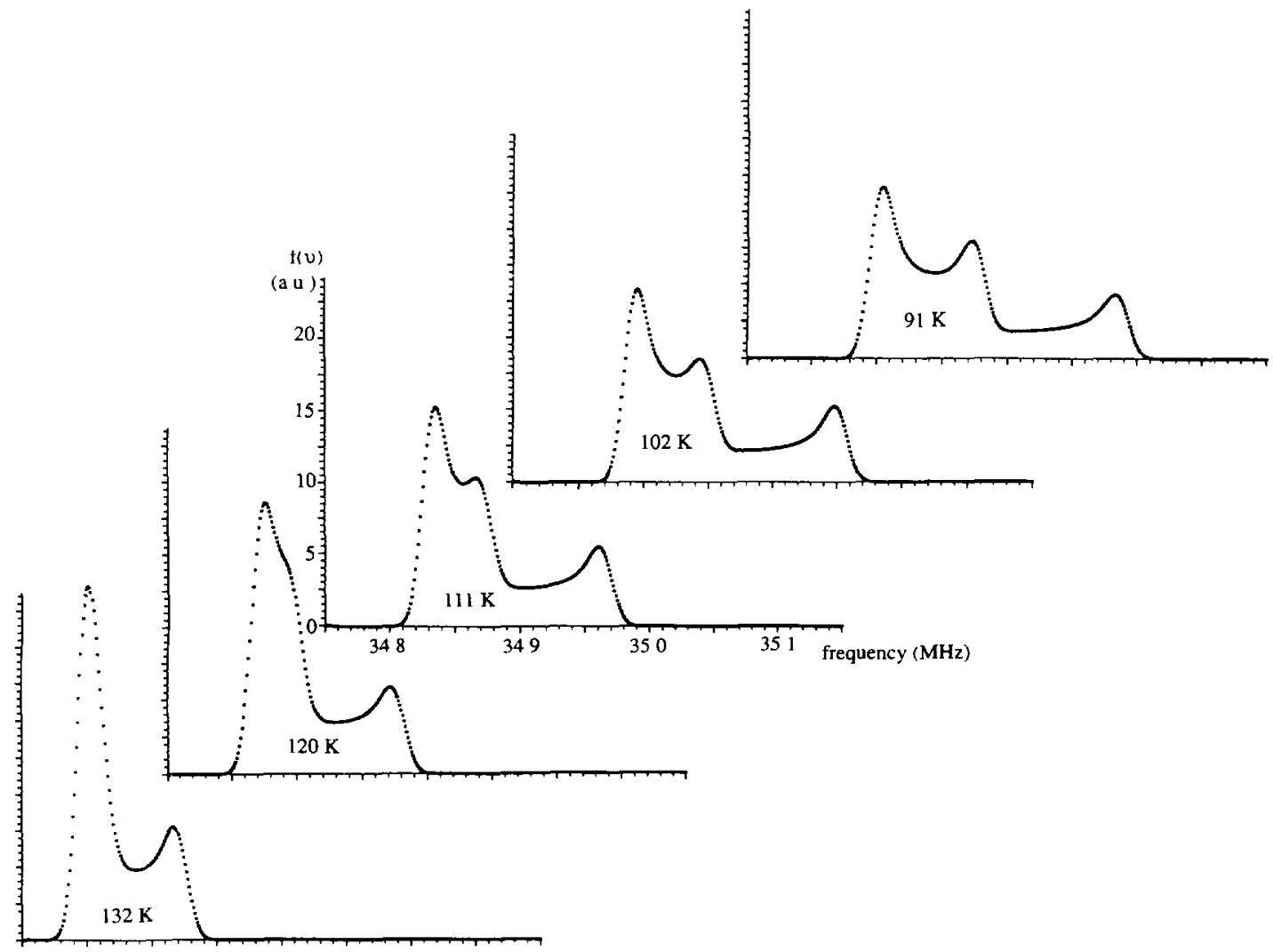

Fig. 7. - Spectral function curves calculated with the parameters derived from the experimental data (see text), including convolution with an $18 \mathrm{kHz}$ half-height wide Gaussian simulating the instrumental response.

regions, corresponding to locked-in phase, separated by a periodic array of narrow « discommensuration » regions. The phase $\Phi(x)$ of the order parameter is then a nonlinear function of the coordinate $x$ which leads to the complexity of the expression (4). The evolution of the spectrum, as temperature is lowered, is expected to be characterized by a progressive increase of the intensity of the maxima associated to the nearly commensurate regions and a simultaneous decrease of the intensity between these maxima [23]. Both models, the crystallographic one, characterized by the presence of high order harmonics and a sinewave modulation, and the discommensuration model, predict the appearance of new lines in the spectrum. NQR measurements at lower temperature would show these differences. Other authors [17] have carried on the ${ }^{35} \mathrm{Cl} \mathrm{NQR}$ study in BCPS down to liquid helium temperature. They observed new lines that they interpreted as characteristic of a low temperature commensurate phase. As it is clear now that there is no lock-in transition in this compound at least down to $4.2 \mathrm{~K}$, a new NQR investigation should be done to continuously follow the spectrum evolution in this whole temperature range. This would precise the deformation of the static incommensurate modulation when going towards a nonsinusoidal regime with the apparition of narrow solitons.

3.3 Spin-lattice Relaxation. - In the temperature dependence of the ${ }^{35} \mathrm{Cl}$ spin-lattice relaxation time, Pusiol et al. [16] found an argument in favour of a lock-in phase transition at 


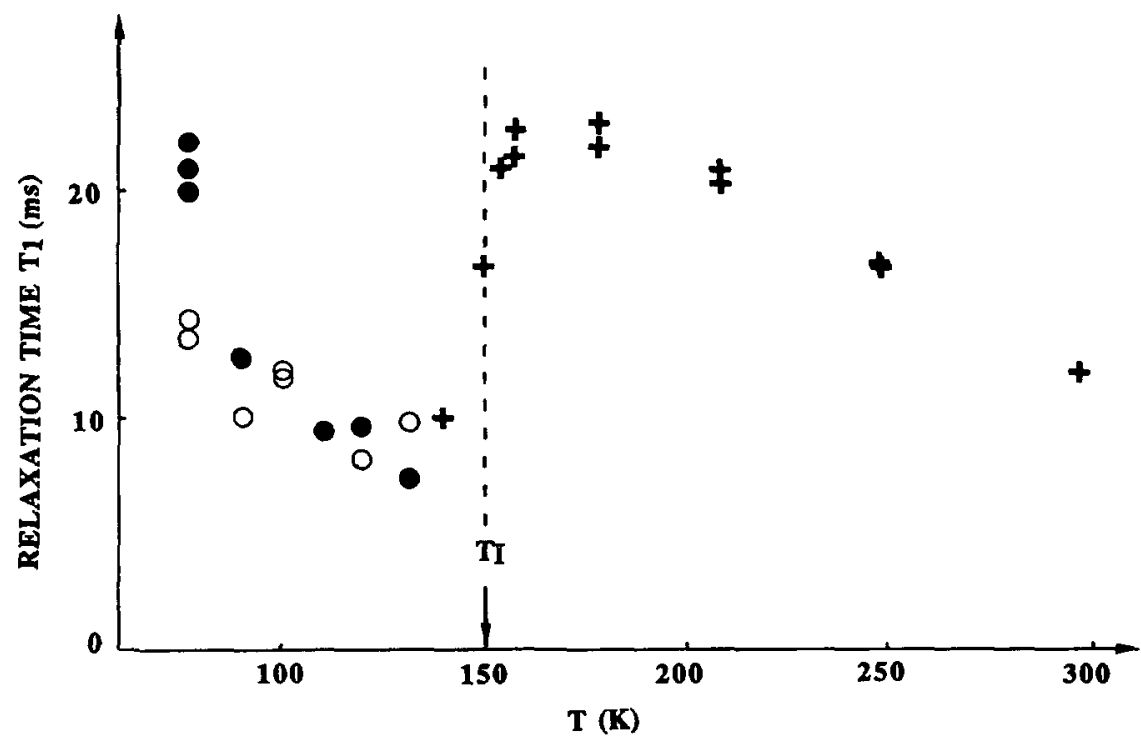

Fig. 8. - Temperature evolution of the spin-lattice relaxation times measured in the high temperature phase $(+)$ on the center of the line, and, in the incommensurate phase, on the low-frequency $(0)$ and highfrequency $(\bullet)$ peaks. In the incommensurate phase, the relaxation is not exponential and the value that can be proposed as characteristic time depends upon the method employed to calculate it; the values shown in the figure are half the time necessary for the signal to recover to $\left(1-1 / e^{2}\right)$ of its full value after a saturation pulse.

$115 \mathrm{~K}$ : they observed a sharp increase in $T_{1}$ below $110 \mathrm{~K}$. This is usually observed when the phason spectrum vanishes at the transition from an incommensurate to a commensurate phase [23]. We made the same kind of measurements as Pusiol and the results are shown in figure 8. In the normal phase, above $150 \mathrm{~K}$, where the spectrum consists of a single line, narrow as compared to the frequency spectrum covered by a saturation pulse, $T_{1}$ values are quite similar to those of Pusiol. Below $150 \mathrm{~K}$, in the incommensurate phase, the spectrum becomes wider and wider; below $140 \mathrm{~K}, T_{1}$ was measured at different frequencies in the spectrum and the values at both ends of the spectrum maxima were recorded. Between $140 \mathrm{~K}$ and $100 \mathrm{~K}$, $T_{1}$ does not much change through the spectrum and remains close to $8 \mathrm{~ms}$; the fact that, between $140 \mathrm{~K}$ and $100 \mathrm{~K}$, the relaxation time $T_{1}$ does not vary appreciably through the spectrum is unexplained in the frame of the usual model of spin-lattice relaxation for quadrupolar nuclei in incommensurate phase [5, 23]. Below $100 \mathrm{~K}, T_{1}$ increases sharply and its value, at $77 \mathrm{~K}$, varies from about $20 \mathrm{~ms}$ on the high frequency peak to $15 \mathrm{~ms}$ on the low frequency peak and $10 \mathrm{~ms}$ in the high frequency valley. These values are only indicative as the relaxation is not exactly exponential in this temperature region and the signal-to-noise ratio is poor in the valley. On the whole, the overall $T_{1}$ behaviour is similar to that reported by Pusiol.

The important increase in $T_{1}$, below $100 \mathrm{~K}$, reveals a modification of the spectral correlation function of the phason branch. As the absence of major change in the spectrum around this temperature precludes the consideration of a lock-in phase transition, another mechanism should be found to explain this modification. It may result from an evolution of the incommensurate structure from a sinusoidal to a soliton regime, as discussed above. Then, theory predicts the opening of gaps in the phason branch in the reciprocal space for the wave vector corresponding to the ordered locked commensurate phase [26]. As the commensurate 
phase has not been observed, this wave vector is not known in BCPS. This makes difficult a quantitative estimation of the effect of the modification of the dynamics on the NMR relaxation processes in this compound. To a first approximation, one may consider that going from a sinusoidal incommensurate dynamics to a soliton-like type yields a decrease of the spectral density of the correlation function. Since this is the efficient process for the nuclear spin-lattice relaxation, it could explain the increase of $T_{1}$ below $100 \mathrm{~K}$ in BCPS.

\section{Conclusion.}

The present $C_{\mathrm{p}}$ investigation in BCPS, from $300 \mathrm{~K}$ down to $90 \mathrm{~K}$, reveals only one transition, displacive and second order at $T_{\mathrm{I}}=150 \mathrm{~K}$. The chlorine-35 NQR spectra recorded from room temperature down to $77 \mathrm{~K}$, confirm the existence of an incommensurate phase below $150 \mathrm{~K}$ and, also, the absence of a lock-in transition in this temperature range. The three peak structure observed in the incommensurate phase and below $140 \mathrm{~K}$, shows the presence of a quadratic term in addition to the linear one in the expression of the resonance frequency in terms of the atomic, or molecular, displacements. These new results, from calorimetry and NQR investigations, are in agreement with those obtained from neutron and $\mathrm{X}$-ray diffraction, the latter methods showing the incommensurate phase persists down to the lowest temperature studied. BCPS provides an exceptional opportunity for studying the dynamics of a simple incommensurate system with very weakly damped excitations when passing from a sinusoidal incommensurate phase to a soliton-like phase. Further NQR investigation, on the temperature dependence of the frequencies and relaxation times, down to liquid helium temperature, would bring forth fundamental informations on static and dynamical aspects of the incommensurate phase in BCPS.

\section{Acknowledgements.}

The authors are grateful to J. Gallier and L. Toupet for useful discussions and Dr. Jakubowski of Institute of Organic and Physical Chemistry (Wroclaw, Poland) for providing the single crystal.

\section{References}

[1] Incommensurate Phases in Dielectrics, in modern problems in condensed matter sciences, vol. 1 and 2, R. Blinc and A. P. Levanyuk Eds. (North Holland, 1986).

[2] Janssen T. and Janner A., Incommensurability in crystals, Adr. Phys. 36 (1987) 519-624.

[3] Currat R. and Janssen T., Excitations in incommensurate crystal phases, Solid State Phys. 41 (1988) 201-302.

[4] Cummins H. Z., Experimental studies of structurally incommensurate crystal phases, Phys. Rep. 185 (1990) 211-411.

[5] Blinc R., Magnetic resonance and relaxation in structurally incommensurate systems, Phys. Rep. 79 (1981) 331-398.

[6] Borsa F. and Rigamonti A., Structural Phase Transition II, K. A. Muller and H. Thomas Eds. (Springer-Verlag, Berlin, 1991) pp. 83-183.

[7] Cailleau H., in [1], vol. 2, chap. 12.

[8] Currat R., Bernard L. and Delamoye P., in [1], vol. 2, chap. 15.

[9] Kasano H., Koshiba T., Kasatani H. and Terauchi H., J Phys. Soc Jpn 59 (1990) 408-411.

[10] Etrillard J., Even J., Sougoti M., Launois P., Longeville S. and Toudic B., Solid. State Commun. 87 (1993) 47-51.

[11] Ishii K.. Nakayama H., Sakato T. and Kano H., Phys. Soc. Jpn 61 (1992) 2317-2326. 
[12] Sougoti M. and Ecolivet C., private communication.

[13] Zuniga F. J., Perez-Mato J. M. and Breczenski T., to be published.

[14] Liu S. B. and Conradi S., Phys. Rev. Lett. 54 (1985) 1287-1290.

[15] De Souza R. E., Engelsberg M. and Pusiol D. J., Phys. Rev. Lett. 66 (1991) 1505-1508.

[16] Pusiol D. J., Wolfenson A. E. and Brunetti A. H., Phys. Rev. B 40 (1989) 2523-2528.

[17] Nakayama H., Eguchi T. and Kishita M., Proceedings of the XIth International Symposium on Nuclear Quadrupole Resonance Spectroscopy, Z. Naturforsch., London 47a (1991) 232-236.

[18] Saito K., Kamio H., Kikuchi K. and Ikemoto I., Thermochim. Acta 163 (1990) 241-248.

[19] Aston J. G., Szasz G. J. and Fink H. L., J. Am. Chem. Soc. 65 (1943) 1135-1143.

[20] Ginnings D. C. and Furakawa G. T., J. Am. Chem. Soc. 75 (1953) 522-527.

[21] Christidès A., Thèse de $3^{e}$ cycle, Orsay, France (1986).

[22] Brown R. J. C., Christidès A., Gourdji M. and Guibé L., J. Mol. Struct. 192 (1989) 355-367.

[23] Blinc R., Prelovsek P., Rutar V., Seliger J. and Zumer S., in [1], vol. 1, chap. 4.

[24] Bourges P., Thèse de l'Université de Rennes, France (1989).

[25] Kusida T., Benedek G. B. and Bloembergen N., Phys. Rev. B 104 (1956) 1364-1377.

[26] Bruce A. D. and Cowley R. A., J. Phys. C 11 (1978) 3609-3630. 